Neurol Med Chir (Tokyo) 50, 505 508, 2010

\title{
Pure Germinoma of the Pineal Gland With Synchronous Spinal Dissemination
}

\section{-Case Report-}

\author{
Masaya NAGAISHI, Ryotaro SUZUKI, Yoshihiro TANAKA, Katumi HOYA, \\ Yoshitaka NARITA*, Aya SHINOMIYA*, Soichiro SHIBUI*, and Akio HYODO \\ Department of Neurosurgery, Koshigaya Hospital Dokkyo University \\ School of Medicine, Koshigaya, Saitama; \\ * Department of Neurosurgery, National Cancer Center Hospital, Tokyo
}

\begin{abstract}
An 11-year-old boy presented with pineal pure germinoma with spinal dissemination manifesting as a 1-month history of ocular motility disturbance and a history of abnormal sensations in the left leg persisting for several months. His past medical history was unremarkable. Craniospinal magnetic resonance imaging showed an enhanced tumor in the pineal gland and widespread leptomeningeal dissemination in the spinal canal. Biopsy of the pineal tumor was performed. Histological examination revealed a pure germinoma. Chemotherapy with carboplatin and etoposide in combination with radiotherapy induced complete remission of the tumors. He regained normal eye movement and sensation in his left leg during the chemotherapy period. Germinomas with dissemination are generally more malignant and refractory than solitary germinomas, but this patient showed a strong response to chemoradiotherapy.
\end{abstract}

Key words: germinoma, dissemination, spine, central nervous system, chemotherapy

Received August 12, 2009; Accepted November 10, 2009

Author's present address: M. Nagaishi, M.D., Department of Human Pathology, Gunma University Graduate School of Medicine, Maebashi, Gunma, Japan. 


\section{Introduction}

Pure germinomas in the central nervous system (CNS) usually occur as a solitary lesion in the pineal or neurohypophyseal region. Multiple or disseminated lesions, including synchronous lesions in the pineal and suprasellar regions, are relatively rare, occurring in only $6-15 \%$ and $4-9 \%$ of whole germ cell tumors, respectively. ${ }^{7,8,11,16,18,21)}$ Multiple or disseminated lesions generally have a poorer prognosis than solitary lesions because of the higher recurrence rate. ${ }^{1,7)}$ Dissemination is usually seen at recurrence after treatment or during the development of nongerminomatous tumors. ${ }^{5,17,18)}$ Pure germinomas with spinal dissemination at the initial diagnosis are rare ${ }^{6)}$ Spinal dissemination is more prevalent in patients with pineal germ cell tumors and tumors that have been histologically classified as germinomas or endodermal sinus tumors. ${ }^{9,24)}$ Additionally, spinal dissemination at diagnosis is rare because the most prevalent locations are intracranial, and dissemination is usually identified using cerebrospinal fluid (CSF) cytology. ${ }^{6,8,11)}$

We describe an unusual case of a pineal pure germinoma that showed spinal dissemination at initial diagnosis.

\section{Case Report}

An 11-year-old boy consulted a neurologist because of progressive disturbance in ocular motility persisting for about one month. Cranial computed tomography demonstrated a large pineal lesion with partial calcification that was causing obstructive hydrocephalus. $\mathrm{T}_{1}$-weighted magnetic resonance (MR) imaging with gadolinium showed a well-enhanced large pineal tumor (Fig. 1A).

On admission, neurological examination revealed limitations in both upward gaze and convergence, slight pupillary dilation, and sluggish light reflex in the right eye. Fundus examination showed bilateral papilledema. In addition, he had complained of abnormal sensations in the left
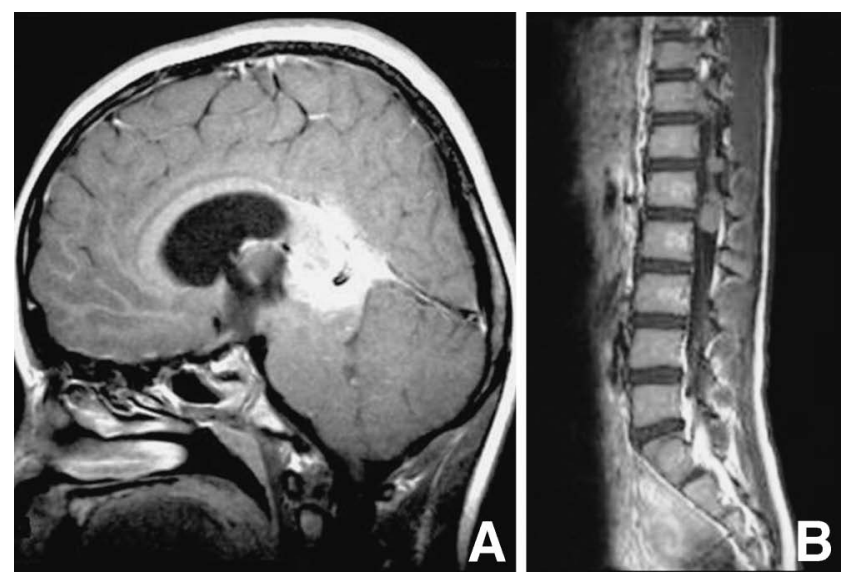

Fig. 1 A: Sagittal $T_{1}$-weighted magnetic resonance (MR) image with gadolinium revealing a homogeneous intensity mass in the pineal region. B: Sagittal $T_{1}$-weighted $M R$ image with gadolinium of the thoracolumbar spine showing multiple slightly enhanced mass lesions from T12 to L2. leg that had worsened during exercise during the past months, so MR imaging with gadolinium was performed which showed multiple slightly enhanced intradural-extramedullary masses at the T12 to L2 levels (Fig. 1B). The serum and CSF levels of beta-human chorionic gonadotropin ( $\beta$-HCG) were slightly elevated at 3.7 $\mathrm{mIU} / \mathrm{ml}$ and $0.2 \mathrm{ng} / \mathrm{ml}$, respectively. In contrast, alphafetoprotein was not detected in either the serum or CSF. CSF cytology revealed no abnormalities.

We suspected a non-germinomatous or mixed type germ cell tumor with leptomeningeal metastasis in the spinal canal based on the MR imaging findings and clinical course. The patient underwent a biopsy using an occipital transtentorial approach to enable histological diagnosis of the pineal lesion. The mass had a grayish bloody appearance with an undefined margin. Frozen section pathological examination identified pure germinoma. Complete removal was not attempted, and the operation was concluded after insertion of a ventricular drain. Histological examination showed a specific "two cell pattern" appearance, and immunohistochemical studies revealed that the tumor cells were stained positively for placental alkaline phosphatase but not for $\beta$-HCG (Fig. 2). The histological diagnosis was pure germinoma.

Following surgery, chemotherapy consisting of carboplatin (525 mg) and etoposide (175 mg) on days 1-3 (CARB-VP regimen) was given for three courses at 4-week intervals. During chemotherapy, nausea and neutropenia occasionally appeared, but were not severe enough to warrant the discontinuation of treatment. After the third cycle of chemotherapy, MR imaging showed that the pineal tumor had been markedly reduced and the leptomeningeal dissemination in the spinal cord had disappeared com-

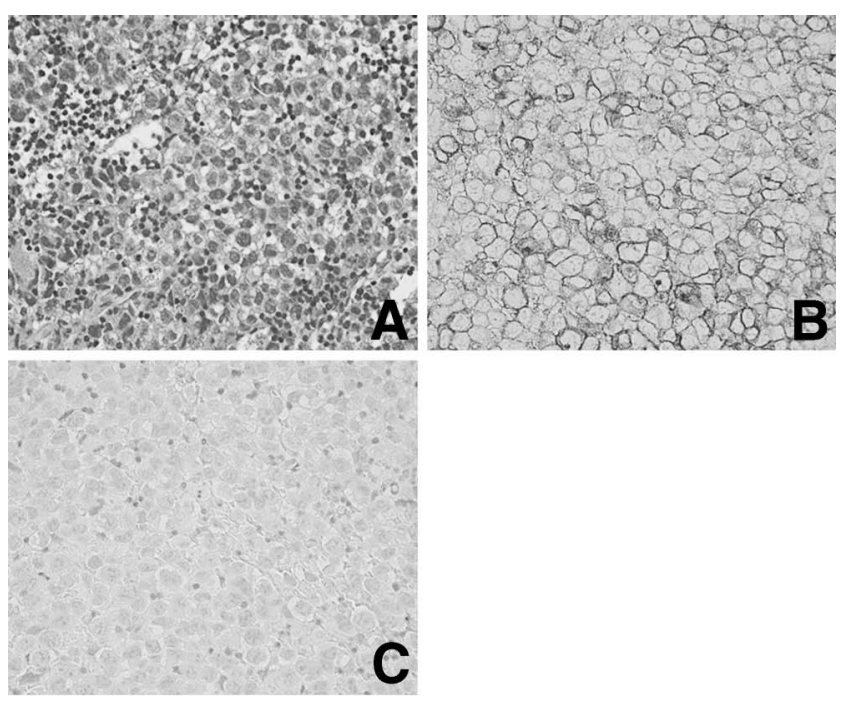

Fig. 2 A: Photomicrograph of the pineal tumor showing a twocell pattern compatible with pure germinoma. Hematoxylin and eosin stain, original magnification $\times 400$. B, C: Immunohistochemical staining for placental alkaline phosphatase demonstrated positive cells (B) but staining for beta-human chorionic gonadotropin was negative (C). Original magnification $\times \mathbf{4 0 0}$. 

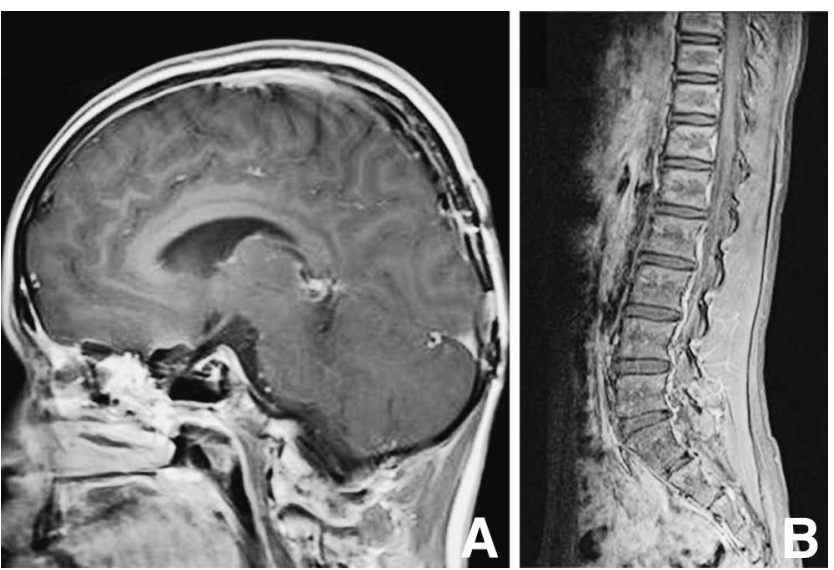

Fig. 3 Intracranial (A) and spinal (B) $\mathrm{T}_{1}$-weighted magnetic resonance images with gadolinium after the completion of therapy showing complete remission of both the pineal tumor and spinal dissemination.

pletely. The patient also regained normal eye movement and sensation in his left leg. Subsequently, radiotherapy was administered to the craniospinal axis at a dose of 30 Gy in 19 fractions over 3 weeks in addition to $20 \mathrm{~Gy}$ in 10 fractions over 2 weeks delivered to a localized field at the pineal tumor site. The daily dose was 1.5 to $2 \mathrm{~Gy}$ for the craniospinal axis and $2 \mathrm{~Gy}$ for the localized field. Subsequent MR imaging of the brain and whole spine detected no tumors (Fig. 3). The patient was able to return to school without experiencing further symptoms. He was asymptomatic, and MR imaging showed no evidence of recurrence at the 8-month follow-up examination.

\section{Discussion}

Chemotherapy results in tumor regression in the majority of patients with pure germinomas. Patients with dissemination at the initial diagnosis responded to chemotherapy as did as those without dissemination. ${ }^{3,15,19)}$ Combination chemotherapy consisting of a cisplatin-etoposide (PE regimen), an ifosfamide-cisplatin-etoposide (ICE regimen), or other similar regimens has generally been used with excellent results. ${ }^{12)}$ However, adjuvant radiotherapy is indispensable because chemotherapy is associated with a higher recurrence rate following salvage treatment. ${ }^{2,14,20,25)}$ In particular, whole CNS irradiation is still recommended as a standard therapy for patients with positive CSF cytology for tumor cells or tumor deposits observed by MR imaging. ${ }^{10,13,21-23)}$ The results of multiple studies have confirmed the efficacy of such combination therapies and the excellent outcome for germinomas. ${ }^{13,16,19,20)}$ However, too few cases of germinomas with dissemination at the initial diagnosis have included detailed descriptions including long-term outcomes to establish an evidence-based treatment regimen.

A phase II trial investigated the response rate and survival of 17 patients treated with conventional doses of etoposide and cisplatin, followed by radiation therapy with dose and volume adjustments according to histologi- cal type and the response to chemotherapy. Patients with disseminated germinoma who achieved complete remission after chemotherapy received $10 \mathrm{~Gy}$ to the local field plus $20 \mathrm{~Gy}$ to the craniospinal axis. Patients with less than complete remission were given $24 \mathrm{~Gy}$ to the local field plus $30 \mathrm{~Gy}$ to the craniospinal axis. Three previously reported patients with germinomas and leptomeningeal spread at the initial diagnosis exhibited complete regression after chemotherapy, and so received lower doses of radiotherapy ${ }^{4)}$ Three patients with CSF dissemination at the initial diagnosis were treated with an ICE regimen of chemotherapy followed by $24 \mathrm{~Gy}$ craniospinal radiotherapy without local boost. ${ }^{19)}$ All six patients achieved complete response after chemoradiotherapy and were free from recurrence after a follow-up period ranging from 6-62 months.

We treated our patient with a CARB-VP regimen as the chemotherapeutic modality based on the results of past studies showing this regimen was effective for germinomas with dissemination. Our patient showed complete response for the spinal lesions, but a small residual tumor remained in the pineal region after chemotherapy. The chemotherapy was followed by whole CNS irradiation (30 Gy) with a local boost to the pineal region (20 Gy), and subsequent MR imaging showed complete response for all lesions. The treatment response in our patient was as strong as those in patients with solitary germinoma, and no differences in clinical or histological malignancy were observed. However, previous clinical trials did not report long-term observations, and no consistent conclusions regarding the optimal treatment have been reached. Longterm outcomes, including relapse and the side effects of radiotherapy, must be assessed to standardize the treatment of germinomas with dissemination at the initial diagnosis. ${ }^{8)}$

\section{References}

1) Allen JC, DaRosso RC, Donahue B, Nirenberg A: A phase II trial of preirradiation carboplatin in newly diagnosed germinoma of the central nervous system. Cancer 74: 940-944, 1994

2) Balmaceda C, Heller G, Rosenblum M, Diez B, Villablanca JG, Kellie S, Maher P, Vlamis V, Walker RW, Leibel S, Finlay JL: Chemotherapy without irradiation-a novel approach for newly diagnosed CNS germ cell tumors: results of an international cooperative trial. J Clin Oncol 14: 2908-2915, 1996

3) Brandes AA, Pasetto LM, Monfardini S: The treatment of cranial germ cell tumours. Cancer Treat Rev 26: 233-242, 2000

4) Buckner JC, Peethambaram PP, Smithson WA, Groover RV, Schomberg PJ, Kimmel DW, Raffel C, O’Fallon JR, Neglia J, Shaw EG: Phase II trial of primary chemotherapy followed by reduced-dose radiation for CNS germ cell tumors. J Clin Oncol 17: 933-940, 1999

5) Diez B, Balmaceda C, Matsutani M, Weiner HL: Germ cell tumors of the CNS in children: recent advances in therapy. Childs Nerv Syst 15: 578-585, 1999

6) Hengstman GJD, Gons RAR, Lenssen PPA, Kappelle AC: Intracranial germinoma presenting as polyradiculopathy due to widespread spinal dissemination. J Neurooncol 80: 105-106, 2006

7) Hoffman HJ, Otsubo H, Hendrick EB, Humphreys RP, Drake 
JM, Becker LE, Greenberg M, Jenkin D: Intracranial germcell tumors in children. J Neurosurg 74: 545-551, 1991

8) Huh SJ, Shin KH, Kim IH, Ahn YC, Ha SW, Park CI: Radiotherapy of intracranial germinomas. Radiother Oncol 38: 19-23, 1996

9) Jennings MT, Gelman R, Hochberg F: Intracranial germ-cell tumors: natural history and pathogenesis. J Neurosurg 63: 155-167, 1985

10) Kumabe T, Kusaka Y, Jokura H, Ikeda H, Shirane R, Yoshimoto T: [Recurrence of intracranial germinoma initially treated with chemotherapy only]. No Shinkei Geka 30: 935-942, 2002 (Jpn, with Eng abstract)

11) Linstadt D, Wara WM, Edwards MSB, Hudgins RJ, Sheline GE: Radiotherapy of primary intracranial germinomas: the case against routine craniospinal irradiation. Int J Radiat Oncol Biol Phys 15: 291-297, 1988

12) Matsutani M; Japanese Pediatric Brain Tumor Study Group: Combined chemotherapy and radiation therapy for CNS germ cell tumors-the Japanese experience. J Neurooncol 54: 311-316, 2001

13) Matsutani M, Sano K, Takakura K, Fujimaki T, Nakamura O, Funata N, Seto T: Primary intracranial germ cell tumors: a clinical analysis of 153 histologically verified cases. J Neurosurg 86: 446-455, 1997

14) Merchant TE, Davis BJ, Sheldon JM, Leibel SA: Radiation therapy for relapsed CNS germinoma after primary chemotherapy. J Clin Oncol 16: 204-209, 1998

15) Neuwelt EA, Wiliams PC, Mickey BE, Frenkel EP, Henner WD: Therapeutic dilemma of disseminated CNS germinoma and the potential of increased platinum-based chemotherapy delivery with osmotic blood-brain barrier disruption. Pediatr Neurosurg 21: 16-22, 1994

16) Ogawa K, Shikama N, Toita T, Nakamura K, Uno T, Onishi H, Itami J, Kakinohana Y, Kinjo T, Yoshii Y, Ito H, Murayama S: Long-term results of radiotherapy for intracranial germinoma: a multi-institutional retrospective review of 126 patients. Int J Radiat Oncol Biol Phys 58: 705-713, 2004

17) Rohr EV, Gönner F, Schroth G, Cerny T: Relapse and subarachnoid dissemination of a pineal germinoma 14 years after radiation therapy. J Clin Neurosci 6: 247-249, 1999

18) Sawamura Y, Ikeda J, Shirato H, Tada M, Abe H: Germ cell tumours of the central nervous system: treatment consideration based on 111 cases and their long-term clinical outcomes. Eur J Cancer 34: 104-110, 1998

19) Sawamura $Y$, Shirato H, Ikeda J, Tada M, Ishii N, Kato T, Abe H, Fujieda K: Induction chemotherapy followed by reduced-volume radiation therapy for newly diagnosed central nervous system germinoma. J Neurosurg 88: 66-72, 1998

20) Schoenfeld GO, Amdur RJ, Schmalfuss IM, Morris CG, Keole SR, Mendenhall WM, Marcus RB: Low-dose prophylactic craniospinal radiotherapy for intracranial germinoma. Int J Radiat Oncol Biol Phys 65: 481-485, 2006

21) Shibamoto Y, Oda Y, Yamashita J, Takahashi M, Kikuchi H, Abe M: The role of cerebrospinal fluid cytology in radiotherapy planning for intracranial germinoma. Int J Radiat Oncol Biol Phys 29: 1089-1094, 1994

22) Shibamoto Y, Takahashi M, Sasai K: Prognosis of intracranial germinoma with syncytiotrophoblastic giant cells treated by radiation therapy. Int J Radiat Oncol Biol Phys 37: 505-510, 1997

23) Shirato H, Nishio M, Sawamura $Y$, Myohjin M, Kitahara T, Nishioka T, Mizutani Y, Abe H, Miyasaka K: Analysis of long-term treatment of intracranial germinoma. Int J Radiat Oncol Biol Phys 37: 511-515, 1997

24) Tosaka $M$, Ogimi $T$, Itoh J, Itoh $H$, Hayashi $S$, Ono $N$, Nagaya T: Spinal epidural metastasis from pineal germinoma. Acta Neurochir (Wien) 145: 407-410, 2003

25) Wolden SL, Wara WM, Larson DA, Prados MD, Edwards MSB, Sneed PK: Radiation therapy for primary intracranial germ-cell tumors. Int J Radiat Oncol Biol Phys 32: 943-949, 1995

Address reprint requests to: Masaya Nagaishi, M.D., Department of Human Pathology, Gunma University Graduate School of Medicine, 3-39-22 Showa-machi, Maebashi, Gunma 371-8511, Japan. e-mail: nagaishi-nsu@umin.ac.jp 